\title{
Assessment of coronary physiology - the evidence and implications
}

\author{
Authors: Noman Ali, ${ }^{A}$ Peysh A Patel ${ }^{B}$ and Christopher J Malkin ${ }^{C}$
}

\begin{abstract}
Use of angiography for the assessment of coronary lesions is limited by its inability to provide information regarding the functional significance of stenoses. A number of studies have demonstrated the presence of ischaemia to be the most important determinant of the benefit associated with coronary revascularisation in stable coronary artery disease. Assessment of intra-coronary physiology can guide percutaneous coronary intervention, and is often used for angiographically borderline stenoses. There is now increasing evidence to suggest that more routine use can improve clinical outcomes. Fractional flow reserve (FFR) is the most established measure of intra-coronary physiology, but is currently underutilised. The main drawback of FFR is the dependence on a pharmacological infusion to maintain hyperaemia. An alternative technique which measures flow at a specific point in the cardiac cycle (instantaneous wave-free ratio) has been developed which obviates the need for hyperaemia and may replace FFR as the default measure.
\end{abstract}

KEYWORDS: Coronary artery disease, angina, percutaneous coronary intervention, coronary physiology, evidence-based medicine

\section{Introduction}

Angina due to coronary artery disease (CAD) constitutes one of the most common medical presentations, both in acute and outpatient settings. While pharmacological therapy remains the cornerstone treatment for stable angina, revascularisation may be necessary where symptoms remain poorly controlled. Anatomical assessment of the coronary vasculature is a necessity in this situation, and invasive angiography is mandatory. However, coronary angiography alone is inherently limited by its inability to provide information pertaining to the functional significance of stenoses. Traditionally, stenoses which reduce the luminal diameter of epicardial coronary vessels by $>70 \%$ are thought to be 'flow-limiting' and thus responsible for myocardial ischemia.

Authors: ${ }^{A}$ specialist registrar in cardiology, Leeds General Infirmary, Leeds, UK; ${ }^{\mathrm{B}}$ specialist registrar in cardiology, Leeds General Infirmary, Leeds, UK; C ${ }^{C}$ Consultant cardiologist, Leeds General Infirmary, Leeds, UK
However, the significance of a lesion is determined not only by its angiographic severity, but also by how much myocardium is being compromised as a result. A number of studies have demonstrated that the presence or absence of ischaemia is the single most important determinant of the benefit associated with coronary revascularisation in the context of stable CAD. ${ }^{1,2}$ Revascularisation in the setting of significant ischaemia is associated with improved outcomes, whereas no improvement is observed when functionally non-significant lesions are treated. As such, identification and quantification of ischaemia should be a prerequisite when considering an invasive management strategy for stable angina.

A number of non-invasive imaging modalities exist which allow for the assessment of inducible ischaemia, such as stress echocardiography, magnetic resonance imaging (MRI) and nuclear myocardial scanning. Each of these has an extensive evidence base, and their use is advocated in international guidelines for functional assessment prior to revascularisation. However, all of these modalities identify regions of myocardial ischaemia and none give lesion specific data within the coronary artery.

The concept of using the trans-stenotic pressure gradient in a diseased coronary artery as a precise measure to guide percutaneous coronary intervention (PCI) has been with us since the 1980s. In a seminal paper, Andreas Gruentzig's group reported that the magnitude of pressure gradient reduction could be used to judge the success of angioplasty. ${ }^{3}$ Subsequently, and in recognition of the fact that identifying physiologically significant stenoses based upon angiography alone can be difficult, fractional flow reserve (FFR) was developed as a technique to enable physiological assessment of coronary lesions.

This short review outlines the principles behind the importance of intra-coronary physiology; the growing evidence base for its use and the latest developments in the field of coronary physiological assessments.

\section{Fractional flow reserve}

FFR expresses the maximum achievable blood flow to the myocardium supplied by a stenotic artery as a fraction of normal maximum flow. As such, it provides an objective measure of the haemodynamic significance of an epicardial stenosis. It can be measured during coronary angiography by passing a specialised guidewire with a specific solid-state sensor at its tip into the coronary vasculature. Once the sensor is advanced distal to the epicardial lesion in question, a pressure reading can be obtained from downstream to the lesion ( $\mathrm{Pd}$ ). A simultaneous pressure reading can be obtained from the tip of the guide catheter, which 


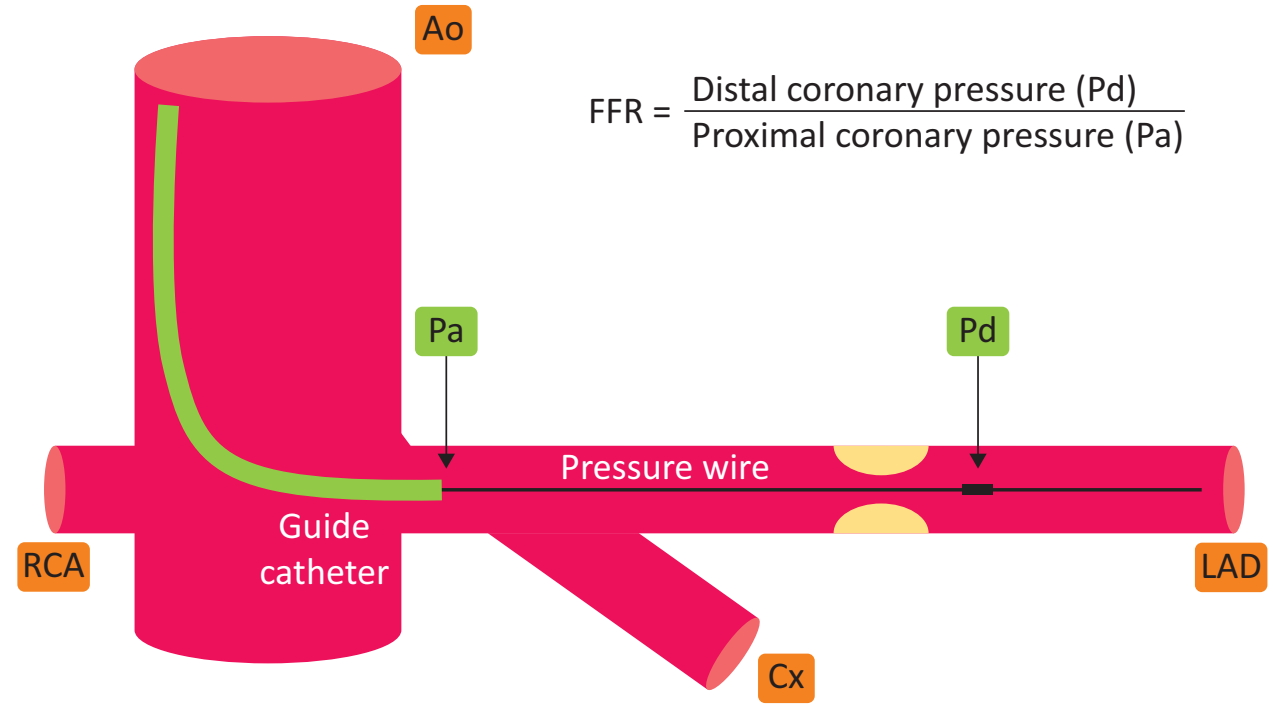

Fig 1. A schematic representation of a pressure wire measurement within the coronary vasculature. The pressure wire is advanced into the coronary artery through a guide catheter, and advanced until the pressure sensor (labelled Pd) is distal to the lesion being assessed. In this example, the fractional flow reserve is being measured across a lesion within the left anterior descending artery. $\mathrm{Ao}=$ aorta; $C x=$ circumflex; $L A D=$ left anterior descending; $\mathrm{Pa}=$ proximal coronary pressure; $\mathrm{Pd}=$ distal coronary pressure; $\mathrm{RCA}=$ right coronary artery. represents the pressure proximal to the lesion ( $\mathrm{Pa})$. By dividing the former by the latter, a ratio of pressures is obtained $(\mathrm{Pd} / \mathrm{Pa}$; see Fig 1). It has been shown that a ratio of flows can be derived from this, provided that the pressures are measured during maximal hyperaemia when resistance is at its lowest. Therefore, one of the most crucial steps in the assessment of FFR is appropriate use of pharmacological hyperaemic stimuli. In order to achieve maximal hyperaemia, vasodilatation of the epicardial and the microvascular circulation is necessary; a bolus of intracoronary nitrate and an intravenous infusion of adenosine respectively are the most commonly used measures.

The initial utility of FFR was to identify angiographic lesions in which PCI was not required. The DEFER (deferral versus performance of percutaneous transluminal coronary angioplasty based on coronary pressure derived fractional flow reserve) study demonstrated the short and long-term safety of deferring PCI for coronary stenoses with an FFR $(\mathrm{Pd} / \mathrm{Pa}) \geq 0.75 .4,5$ Attention then turned to using FFR in order to guide rather than defer PCI; the FAME (fractional flow reserve versus angiography for multivessel evaluation) trial showed that FFR-guided PCI in patients with multivessel coronary disease results in lower 1-year adverse events and reduced costs. ${ }^{6}$ The follow-up FAME- 2 trial went on to highlight the superiority of FFR-guided PCI plus medical therapy compared with medical therapy alone for lesions with an FFR $\leq 0.80{ }^{7}$ This study also highlighted the benefit of not intervening on FFR-negative lesions, where excellent outcomes were seen with medical therapy alone irrespective of angiographic appearance. The FAME data also clearly showed how poor simple angiography was at predicting the functional significance of lesions, even when performed by highly experienced interventional cardiologists. In the group of patients randomised to FFR measurements, a discordance was noted between the operators' angiographic assessment of significant lesions and the FFR result in $37 \%$ of cases; this means that if angiography alone was used, functionally non-significant lesions would have received an inappropriate stent. In clinical practice, the converse can also be true; if revascularisation decisions are made purely on angiographic findings, lesions which are functionally significant but do not appear severe may not be treated. Based upon this body of evidence (see supplementary material S1), use of FFR is advocated in both the current European Society of Cardiology (ESC) and American College of Cardiology Foundation / American Heart Association guidelines for myocardial revascularisation. , $^{89}$

In current clinical practice, FFR is mainly used to assess lesions which are angiographically borderline. In these circumstances, FFR assessment provides a useful objective measure to guide the decision-making process with regard to selecting between medical therapy and revascularisation. The evolving data suggests more routine use of pressure wire assessment, including lesions which appear to be clearly angiographically significant. Every interventional cardiologist will regularly assess coronary stenoses that appear anatomically severe but are in fact functionally not significant, whereas other stenoses which appear mild are proven to be functionally significant when assessed by FFR (see Fig 2).

\section{Growing evidence for FFR}

In the last 18 months, there have been two published sub-studies from FAME-2 which provide increasingly robust evidence for more routine use of FFR (see Table 1 Supplement S1). The first paper, which provides the 3-year follow-up data, highlights two key findings; major adverse cardiac events (MACE; defined as death, myocardial infarction and urgent revascularisation) were lower in the PCI group, and the initial higher cost associated with PCI compared with medical therapy equalised over the course of the follow-up period. ${ }^{10}$ The second paper was perhaps even more interesting, and focused on the subset of patients from FAME-2, in whom no revascularisation was undertaken. ${ }^{11}$ These 607 patients were separated into four groups based upon the angiographic (diameter stenosis (DS)) and FFR-based characteristics of their coronary lesions; positive concordance (FFR $\leq 0.80$; DS $\geq 50 \%$ ), negative concordance (FFR $>0.80$; $D S<50 \%$ ), positive mismatch (FFR $\leq 0.80 ; \mathrm{DS}<50 \%$ ) and negative mismatch (FFR $>0.80$;

$\mathrm{DS} \geq 50 \%$ ). The groups were compared with respect to a composite end point of cardiac death, vessel-related myocardial infarction, and vessel-related revascularisation. Predictably, the study found 

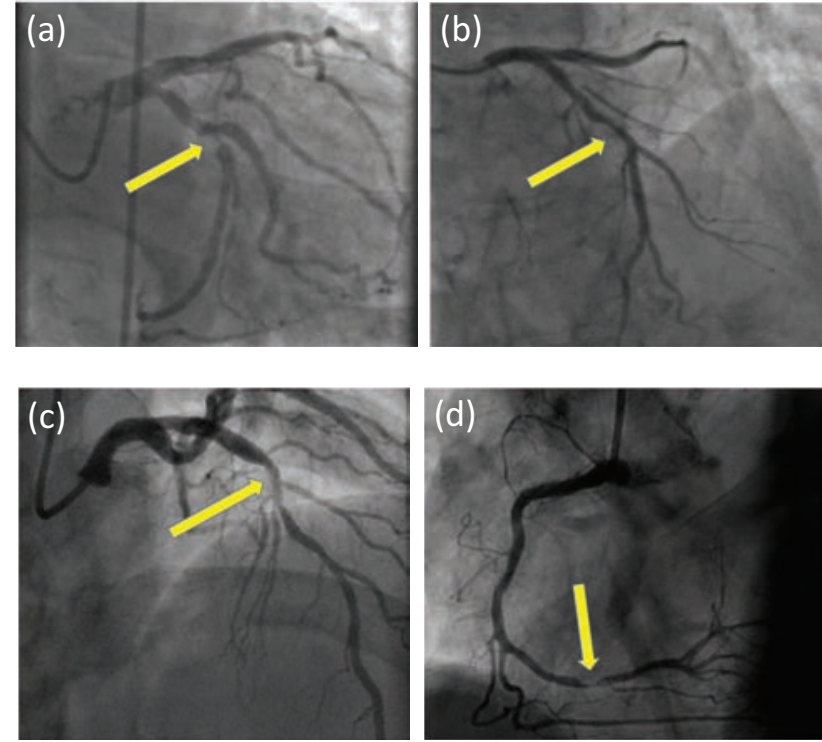

Fig 2. Examples of coronary lesions assessed using fractional flow reserve (FFR). Only two of these lesions are functionally significant.

(a) Angiographically significant stenosis in the circumflex; negative on pressure wire assessment (FFR 0.86); medically managed. (b) Angiographically mildmoderate stenosis in mid-left anterior descending (LAD); positive on pressure wire assessment (FFR 0.76); treated with percutaneous coronary intervention (PCI). (c) Angiographically mild-moderate stenosis in mid-LAD; negative on pressure wire assessment (FFR 0.84); medically managed. (d) Angiographically significant stenosis in distal right coronary artery; positive on pressure wire assessment (FFR 0.74); treated with PCI.

positive concordance to be associated with the worst outcomes, negative concordance to be associated with the best outcomes, and the positive mismatch group to have poorer outcomes than the negative mismatch group. More interesting, however, were the findings of no difference between the positive concordance (FFR $\leq 0.80$; DS $\geq 50 \%$ ) and positive mismatch (FFR $\leq 0.80$; DS $<50 \%$ ) groups, and no difference between the negative mismatch (FFR $>0.80 ; D S \geq 50 \%$ ) and negative concordance (FFR $>0.80$; $\mathrm{DS}<50 \%$ ) groups. These results substantiate the concept that the physiological impact of lesions is a more important determinant of outcome than the angiographic appearance.

\section{Challenges with FFR}

The use of FFR to guide intervention in the context of stable CAD is now well established. However, its utility in the context of acute coronary syndromes (ACS) remains contentious. Patients presenting with ACS often display multivessel disease, and the use of FFR to assess non-culprit lesions has obvious theoretical appeal. However, use of FFR is dependent upon achieving maximal hyperaemia in the vascular bed distal to the coronary lesion being measured, thereby producing a near-linear relationship between pressure and flow. One of the concerns regarding the use of FFR in ACS patients is that the process of plaque rupture and thrombosis formation creates a milieu of vasoactive factors which may preclude an adequate hyperaemic response during the test. Nevertheless, there have been recent studies which suggest that use of FFR may be viable in this setting. The DANAMI-3-PRIMULTI (the third Danish study of optimal acute treatment of patients with STEMI: primary PCI in multivessel disease) trial demonstrated that FFR-guided staged complete revascularisation during the index admission led to a reduction in the primary composite end-point compared with culprit-lesion only treatment. ${ }^{12}$ Similarly, the Compare-acute (fractional flow reserve-guided multivessel angioplasty in myocardial infarction) trial reported that FFR-guided revascularisation at the time of primary PCI was associated with a lower rate of a composite cardiovascular events at 1 year. ${ }^{13}$ While these results are ostensibly encouraging, it must be borne in mind that in both cases the positive findings were driven by a reduction in future revascularisation rather than mortality or myocardial infarction. As such, the evidence base for the use of FFR in the context of ACS remains a work in progress rather than providing a compelling indication.

Another grey zone with respect to the use of FFR is the assessment of serial stenoses in the same vessel. In arteries with multiple significant lesions, there is interaction between the stenoses such that the first stenosis limits maximal flow across downstream lesions and the downstream lesions limit maximal flow across the proximal lesion. Experimental studies have provided a calculation which allows this problem to be circumvented..$^{14}$ However, in order for the calculation to be made, a coronary occlusion wedge pressure during maximal hyperaemia needs to be measured, making it impractical. The more pragmatic approach in the setting of serial stenoses is to perform a pullback of the pressure wire during sustained maximal hyperaemia, assessing for pressure step-ups across the lesions in question. Using this method, the stenosis which generates the largest pressure gradient can be identified and should be treated first. A pressure wire assessment can then be repeated for the remaining stenosis, and PCI performed if the FFR remains $<0.80$.

\section{Instantaneous wave-free ratio - a game-changer?}

Despite the ever-increasing evidence base for the use of FFR in stable CAD, its usage remains disappointingly low. ${ }^{15}$ One of the barriers associated with its use is the requirement for an infusion of adenosine in order to achieve maximal hyperaemia. This adds time and complexity to the procedure, as well as exposing patients to adverse side effects including chest pain, shortness of breath and flushing. FFR is also limited to an extent in that tandem lesions or true bifurcation lesions can be difficult to interrogate. In recent years, the development of instantaneous wave-free ratio (iFR) has been seen as a breakthrough in the field of coronary intervention. The technique is centred around the concept that at a specific point in diastole, referred to as the wave-free period, flow and pressure within the coronary arteries are linearly related. The wave-free period is defined as beginning from $25 \%$ into diastole to $5 \mathrm{~ms}$ before the end of diastole, and measurement of a pressure gradient across a stenosis during this time obviates that need to generate hyperaemia, and thus the need for adenosine. Another useful advancement with iFR is the ability to individually assess lesions within diffusely diseased arteries, thus mitigating the difficulty faced by FFR in the setting of serial stenoses as described above. With the development of co-registration, where software can plot measured values directly onto the angiogram, iFR can provide lesion specific data, which is a huge advantage in patients with complex coronary disease. It allows the cardiologist not only to identify which lesion should be treated, but gives accurate data on how much this will improve the physiology and even how long a stent to choose (see Fig 3). 


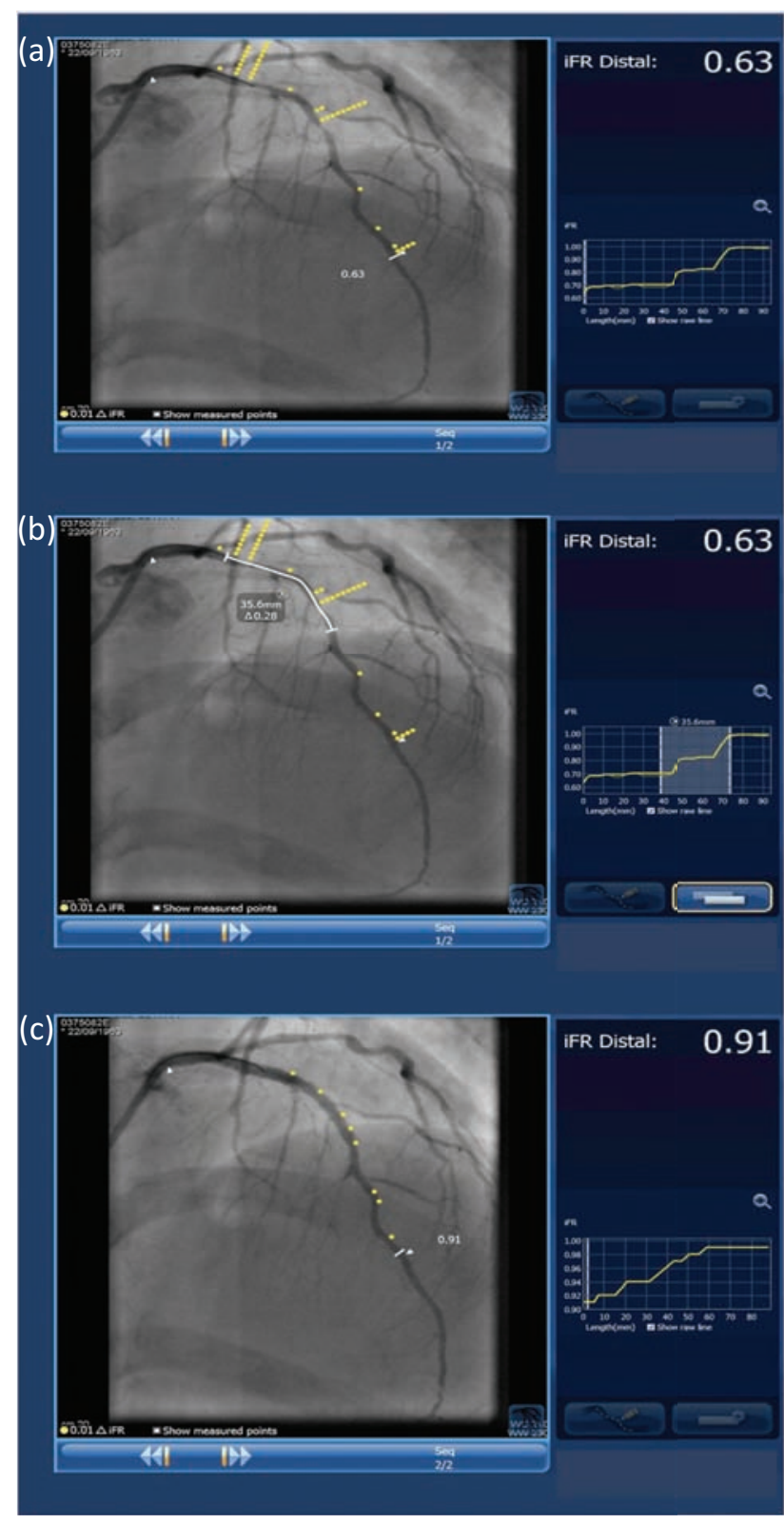

Fig 3. Examples of instantaneous wave free ratio (iFR) with coregistration being used to guide percutaneous coronary intervention (PCI) to the left anterior descending (LAD) artery. The cut-off for iFR is 0.89 , with functionally significant disease defined as iFR $<0.89$. Co-registration plots yellow dots on the angiographic image and they represent the severity of the lesions, with a greater number of dots correlating with a more severe stenosis. Images provided with permission from Dr Bagnall and Philips IGTD. (a) The initial iFR measurement shows multiple lesions within the LAD, with the most severe being in the proximal vessel. The iFR in the distal vessel measures 0.63 , indicating functionally significant disease. (b) ifR with co-registration can be used to measure lesion length and plan stent size. In this example, a stent measuring at least $36 \mathrm{~mm}$ is required. (c) iFR carried out following stent deployment shows a significant improvement in vessel flow, with the post-PCI iFR measuring 0.91 in the distal vessel.

A number of studies have demonstrated correlation between FFR and iFR with regard to diagnostic accuracy. ${ }^{16-18}$ In 2017, two landmark papers were published which highlighted iFR-guided PCI to be non-inferior to FFR-guided PCI with regard to major adverse cardiac events at 1 year. ${ }^{19,20}$ These data have led to increased uptake of iFR by interventional cardiologists, and it is possible that it could replace FFR as the default physiological measure of coronary lesions in years to come.

Given the clear appeal of hyperaemia-independent coronary physiological measures, it is no surprise that the success of iFR has led to the development of other, similar indices. The resting full-cycle ratio (RFR) uses the mean resting $\mathrm{Pd} / \mathrm{Pa}$ over the entire cardiac cycle and has been validated as diagnostically equivalent to iFR in a recent study. ${ }^{21}$ Its advantage over iFR is its simplicity, in that it does not require detection of a specific point in the cardiac cycle. However, RFR-guided PCI has yet to be tested in a randomised clinical trial, and so it cannot yet be considered as a potential replacement for FFR.

\section{Coronary flow reserve and absolute coronary blood flow}

While the use of FFR and iFR provide valuable diagnostic information and allow interventional cardiologists to make more informed decisions regarding the treatment of coronary artery disease, it is important to appreciate the limitations of the technology. The fundamental principle used in both modalities is that pressure measurements can be used to derive an estimate of coronary flow impairment due to a stenotic lesion. The relationship between coronary pressure and flow is not a linear one, and is affected by the resistance of the vessel and the downstream microvasculature. In an attempt to mitigate this effect, vasodilating pharmacological agents such as adenosine are used in order to generate maximal hyperaemia (ie minimal vascular resistance) during FFR measurements. Meanwhile, iFR is calculated during the diastolic wave-free period as a surrogate of maximal hyperaemia since coronary resistance is thought to be constant at this time. Nevertheless, both of these methods are based upon assumptions of equivalence between pressure and flow. Coronary flow reserve (CFR) is a direct measure of coronary blood flow, and has been validated in a number of nonrandomised studies. ${ }^{22}$ CFR is calculated as the ratio of coronary blood during maximal vasodilation divided by coronary blood flow during resting conditions, and can be measured using a thermodilution technique or assessment of Doppler flow velocity. Since coronary flow is more clinically relevant than perfusion pressure in the context of stenotic disease, CFR is a technique of great potential.

Another limitation of FFR and iFR is that they focus upon disease in the epicardial arteries. While lesions in these vessels are potentially life-threatening and amenable to treatment, these arteries represent only around $10 \%$ of total coronary circulation; the other $90 \%$ is comprised of the microvasculature. ${ }^{23}$ Use of the thermodilution technique allows for quantification of absolute coronary blood flow, and may provide an insight into the pathogenesis of microvascular angina, where patients have classical anginal symptoms and demonstrable inducible ischaemia in the absence of functionally significant epicardial stenoses.

It is interesting to note that CFR in fact pre-dates FFR. In spite of this, it is FFR that has been taken up as the tool of choice in real-world practice. CFR measurements are more technically challenging and time-consuming, thus limiting its applicability. Nevertheless, absolute coronary blood flow remains an exciting future target for physiological assessment. 


\section{Conclusion}

It is now becoming accepted that the physiological impact of coronary stenoses has more clinical significance than angiographic severity. The evidence for use of FFR to guide interventional strategy in patients with stable coronary disease is now robust, while data pertaining to its use in the context of ACS is promising. With new techniques, such as iFR enabling pressure wire measurements to be made without use of adenosine, the traditional barriers that existed to the widespread use of FFR can now be circumvented. The question is, will the combination of increasing evidence and developing technology lead to a tangible change in practice whereby physiological measurements prior to revascularisation become routine, or will we remain beholden to the 'occulo-stenotic reflex'?

\section{Supplementary material}

Additional supplementary material may be found in the online version of this article at http://www.clinmed.rcpjournal.org:

S1 - Table summarising the landmark trials using fractional flow reserve and instantaneous wave-free ratio.

\section{References}

1 Hachamovitch R, Hayes SW, Friedman JD, Cohen I, Berman DS Comparison of the short-term survival benefit associated with revascularization compared with medical therapy in patients with no prior coronary artery disease undergoing stress myocardial perfusion single photon emission computed tomography. Circulation 2003;107:2900-7.

2 Hachamovitch R, Rozanski A, Shaw LJ et al. Impact of ischaemia and scar on the therapeutic benefit derived from myocardial revascularization vs medical therapy among patients undergoing stress-rest myocardial perfusion scintigraphy. Eur Heart ] 2011;32:1012-24.

3 Anderson HV, Roubin GS, Leimgruber PP et al. Measurement of transstenotic pressure gradient during percutaneous transluminal coronary angioplasty. Circulation 1986;73:1223-30.

4 Bech GJ, De Bruyne B, Pijls NH et al. Fractional flow reserve to determine the appropriateness of angioplasty in moderate coronary stenosis: a randomized trial. Circulation 2001;103:2928-34.

5 Zimmermann FM, Ferrara A, Johnson NP et al. Deferral vs. performance of percutaneous coronary intervention of functionally nonsignificant coronary stenosis: 15-year follow-up of the DEFER trial. Eur Heart ] 2015;36:3182-8.

6 Tonino PA, De Bruyne B, Pijls NH et al. Fractional flow reserve versus angiography for guiding percutaneous coronary intervention. N Engl J Med 2009;360:213-24.

7 De Bruyne B, Pijls NH, Kalesan B et al. Fractional flow reserveguided PCI versus medical therapy in stable coronary disease. N Engl J Med 2012;367:991-1001.

8 Kolh P, Windecker S, Alfonso F et al. 2014 ESC/EACTS Guidelines on myocardial revascularization: The Task Force on Myocardial Revascularization of the European Society of Cardiology (ESC) and the European Association for Cardio-Thoracic Surgery (EACTS). Developed with the special contribution of the European Association of Percutaneous Cardiovascular Interventions (EAPCI). Eur J Cardiothorac Surg 2014;46:517-92.

9 Levine GN, Bates ER, Blankenship JC et al. 2011 ACCF/AHA/SCAI Guideline for Percutaneous Coronary Intervention: a report of the American College of Cardiology Foundation/American Heart Association Task Force on Practice Guidelines and the Society for Cardiovascular Angiography and Interventions. Circulation 2011:124: e574-651.
10 Fearon WF, Nishi T, De Bruyne B et al. Clinical outcomes and cost-effectiveness of fractional flow reserve-guided percutaneous coronary intervention in patients with stable coronary artery disease: three-year follow-up of the FAME 2 Trial (Fractional Flow Reserve Versus Angiography for Multivessel Evaluation). Circulation 2018:137:480-7.

11 Ciccarelli G, Barbato E, Toth $\mathrm{G}$ et al. Angiography versus hemodynamics to predict the natural history of coronary stenoses: fractional flow reserve versus angiography in multivessel evaluation 2 substudy. Circulation 2018;137:1475-85.

12 Engstrøm T, Kelbæk H, Helqvist S et al. Complete revascularisation versus treatment of the culprit lesion only in patients with ST-segment elevation myocardial infarction and multivessel disease (DANAMI-3—PRIMULTI): an open-label, randomised controlled trial. Lancet 2015;386:665-71.

13 Smits PC, Abdel-Wahab M, Neumann FJ et al. Fractional flow reserve-guided multivessel angioplasty in myocardial infarction. N Engl J Med 2017;376:1234-44.

14 Pijls NH, De Bruyne B, Bech G] et al. Coronary pressure measurement to assess the hemodynamic significance of serial stenoses within one coronary artery: validation in humans. Circulation 2000;102: 2371-7.

15 Zack CJ, Bove AA, Bashir R et al. National utilization rates of fractional flow reserve in guiding coronary revascularization. Abstract presented at AHA, 2012. http://circ.ahajournals.org/cgi/content/ A18105.

16 Escaned J, Echavarría-Pinto M, Garcia-Garcia HM et al. Prospective assessment of the diagnostic accuracy of instantaneous wavefree ratio to assess coronary stenosis relevance: Results of ADVISE II international, multicenter study (ADenosine Vasodilator Independent Stenosis Evaluation II). JACC Cardiovasc Interv 2015;8:824-33.

17 Petraco R, Al-Lamee R, Gotberg M et al. Real-time use of instantaneous wave-free ratio: Results of the ADVISE in-practice: An international, multicenter evaluation of instantaneous wave-free ratio in clinical practice. Am Heart ] 2014;168:739-48.

18 Park J], Petraco R, Nam CW et al. Clinical validation of the resting pressure parameters in the assessment of functionally significant coronary stenosis; results of an independent, blinded comparison with fractional flow reserve. Int J Cardiol 2013;168:4070-5.

19 Davies JE, Sen S, Dehbi HM et al. Use of the instantaneous wave-free ratio or fractional flow reserve in PCI. N Engl ] Med 2017:376:1824-34

20 Götberg M, Christiansen EH, Gudmundsdottir IJ et al. Instantaneous wave-free ratio versus fractional flow reserve to guide PCI. N Engl J Med 2017;376:1813-23.

21 Svanerud J, Ahn JM, Jeremias A et al. Validation of a novel non-hyperaemic index of coronary artery stenosis severity: the resting full-cycle ratio (VALIDATE RFR) study. EuroIntervention 2018;14:806-14.

22 Stegehuis VE, Wijntjens GW, Piek J] et al. Fractional flow reserve or coronary flow reserve for the assessment of myocardial perfusion. Curr Cardiol Rep 2018;20:77.

23 Kanaji Y, Murai T, Yonetsu T et al. Effect of elective percutaneous coronary intervention on hyperhemic absolute coronary blood flow volume and microvascular resistance. Circ Cardiovasc Interv 2017;10:e005073.

Address for correspondence: Dr Noman Ali, Department of Cardiology, Leeds General Infirmary, Leeds LS1 3EX, UK. Email: nomanali456@doctors.org.uk 